\title{
Soziologie in China
}

\author{
Dominique Schirmer
}

Angenommen: 22. Februar 2021 / Online publiziert: 26. März 2021

(C) Der/die Autor(en) 2021

Chen, Hon Fai: Chinese Sociology. State-Building and the Institutionalization of Globally Circulated Knowledge. Cham: Palgrave Macmillan 2018. 149 Seiten. ISBN: 978-1-137-58219-5. Preis: $€ 58,84$.

„Chinese Sociology“ von Chen Hon Fai erscheint in der Reihe „Sociology Transformed“ der Herausgeber John Holmwood und Stephen Turner. Die Serie soll den Wandel der Soziologie(n) in unterschiedlichen Ländern, nicht nur in den Zentren, aufzeigen und damit zu einem globalen Diskurs um das Fach beitragen. Bislang sind Bände zu den Soziologien in 21 Ländern erschienen, von denen gut die Hälfte europäisch ist.

Chen Hon Fai, der an der Lingnan-Universität in Hongkong forscht und lehrt, präsentiert mit „Chinese Sociology“ einen wissens- und wissenschaftssoziologischen Blick auf die Soziologie der Volksrepublik China. Vorneweg: Das Buch gibt einen interessanten und überwiegend kurzweiligen Einblick in die Welt der chinesischen Soziologie. Der - zweideutige - Untertitel „State-Building and the Institutionalization of Globally Circulated Knowledge“ beschreibt Chens Kernthese sowie den roten Faden. Eine Kernthese ist der Einfluss der chinesischen Regierung auf die Entwicklung und die Themen des Faches. Das „State-Building“ spielt allerdings nur in zwei Phasen eine Rolle, zum Beginn des 20. Jahrhunderts sowie insbesondere in den 1980er- und 1990er-Jahren, als gesellschaftliche Umbrüche und die Konsolidierung des Staates für das Fach prägend waren und übrigens auch vom Fach geprägt wurden. Die „Institutionalisierung“ weist insofern auf das Hauptaugenmerk des Werks, als dass Chen sich insbesondere für die Rolle der Institutionen des Fachs

D. Schirmer $(\bowtie)$

Institut für Soziologie, Albert-Ludwigs-Universität Freiburg

Postfach, 79085 Freiburg im Breisgau, Deutschland

E-Mail: dominique.schirmer@soziologie.uni-freiburg.de 
interessiert. Es gibt aber noch ein zweites Schwerpunktthema, das etwa zwei Drittel des Buches umfasst: es zeigt die Produktion und Transformation soziologischen Wissens an ausgewählten Gegenständen.

Im ersten Drittel des Buches fokussiert der Autor die institutionelle Rahmung. Chen beschreibt die Soziologie oder soziologisches Wissen als Produkt ihrer institutionellen Einbettung in intellektuelle Gemeinschaften, die vom Staat, von den Universitäten, Forschungsinstituten, Berufsverbänden und weiteren akademischen Institutionen geformt werden (S. 4). Dabei schreibt Chen der staatlichen Politik den zentralen Einfluss zu (S. 5). Tatsächlich beeinflussen aber beide, die staatliche Politik und die soziologischen Institutionen, das Fach und seine Entwicklung wesentlich und der Einfluss des Staates ist nicht ausschließlich, nicht eindeutig und nicht unidirektional. Zudem kommen der Einfluss der Gesellschaft und des gesellschaftlichen Wandels sowie die Rolle der Soziologen und Soziologinnen als Gesellschaftsmitglieder, insbesondere um die Jahrtausendwende, in Chens Perspektive nicht vor.

$\mathrm{Zu}$ den Inhalten: Nach einer kurzen Einleitung behandelt Chen in Kapitel 2 die Entwicklung und Etablierung der Soziologie um die Jahrhundertwende (vom 19. zum 20. Jahrhundert) bis zur Gründung der Volksrepublik China 1949. Beginnend im kaiserlichen China war diese Zeit geprägt von einem konflikthaften Verhältnis zum Westen, der Gründung der Republik China, von Kriegen und Bürgerkriegen. Chen zeigt die Bemühungen der Institutionalisierung des Faches Soziologie und der Entwicklung und Standardisierung soziologischen Wissens. Auf methodischer Seite sind die großen Umfragestudien in den 1920er-Jahren hervorzuheben sowie eine marxistische Version soziologischer Theorie und Sozialforschung, die sich seit den 1920er-Jahren entwickelt und die eine spezifische Sozialforschung (,shehui diaocha“) betrieben hat. In den 1930er-Jahren beginnt die Ära der großen anthropologischen Feldstudien, der Gemeindestudien, deren berühmtester Forscher Fei Xiaotong war. Er veröffentlichte 1939 „Peasant Life in China“ und 1947,Erdverbundenes China“ (Xiangtu Zhongguo), die zu den prägenden soziologischen Arbeiten gehören.

Kapitel 3 beginnt mit den institutionellen Entwicklungen der Soziologie nach der Wiedereinrichtung des Fachs, nachdem es von 1953-1979 abgeschafft und verboten war. Chen verliert über diese Zeit leider kaum eine Zeile, was wohl der institutionellen Perspektive seiner Fragestellung geschuldet ist. Die Verbotszeit war aber nicht nur prägend bis zum Ende des Jahrtausends, sondern auch auf theoretischer und insbesondere auf methodischer Ebene sowie auf der Ebene des Fachverständnisses bedeutend. Immerhin erwähnt der Autor kurz die soziologischen Aktivitäten (,,intellectual activities“) dieser Zeit in Form von „Soviet-style ethnology, census and rural survey“ (S. 31). Die damalige Sozialforschung bezeichnet er treffend als ,,sort of amateur sociology“. Detailliert setzt sich Chen mit den institutionellen Rahmungen der Entwicklung des Fachs in den zwanzig Jahren bis zur Jahrtausendwende auseinander (und konterkariert damit häufig die Betonung des einseitigen Einflusses der Regierungspolitik). Im Laufe des Kapitels ändert sich der Fokus von einem vor allem institutionellen Blick auf einen vor allem inhaltlichen. Nach der ausführlichen Beschreibung der strategisch vorangetriebenen Wiedereinführung des Faches diskutiert Chen die Entwicklung soziologischer Forschungsparadigmen, gibt aber meines Erachtens der Komplexität des Themas nicht genügend Raum. So bleibt die Verwobenheit von „marxistisch“ oder „maoistisch“, „westlich“ oder „chinesisch“, 
„traditionell“ oder „modern“ der empirischen Forschungsansätze unklar. Deutlich wird aber auch hier die - immer wiederkehrende - Auseinandersetzung zwischen quantitativen und qualitativen Forschungsansätzen.

Im vierten und fünften (und zum Teil im sechsten) Kapitel widmet sich Chen intensiv den inhaltlichen Entwicklungen und Auseinandersetzungen des Fachs. Er behandelt Forschungsthemen und ihren Wandel in soziologischen Diskursen, Theorien und Schulen. Schwerpunkt sind die 1990er- und 2000er-Jahre. Chen fokussiert besonders die Forschungen zu sozialem Wandel, Methodologie, Gender Studies und zur Konsumsoziologie. Zentrale soziologische Themen um die Jahrtausendwende sind soziale Beziehungen, soziales Handeln, Sozialverhalten, soziale Ungleichheit, soziale Schichtung, soziologische Klassiker, Forschungen zur Mittelschicht und Gemeindestudien. Chen diskutiert den Paradigmenwechsel in der Forschung zur sozialen Schichtung und zum sozialen Wandel, die weitreichende Konsequenzen für soziologische Forschung und Theoriebildung hatten (S. 53 f.), sowie den Paradigmenwechsel von Modernisierungstheorien zu Theorien ökonomischer Transformationsprozesse, der Marktwirtschaft und der Ökonomisierung. Außerdem zeichnet der Autor die Positionen von Frauen-, Gender- und feministischen Theorien und Studien nach, den Wandel von der Lebensstil- zur Konsumsoziologie und den Wandel zur postpositivistischen Methodologie. Diese Entwicklungen zeigen die enorme Diversifizierung der chinesischen Soziologie (S. 83). Ebenso zeichnet Chen die Verflechtung und den Austausch von Forschungsansätzen mit gesellschaftlichen und internationalen Akteuren und Akteurinnen, beispielsweise in der feministischen Forschung, nach und führt Beispiele für eine Public Sociology an, die, unter anderem inspiriert von der Hongkonger Soziologin Pun Ngai, soziologische Interventionen in den Fokus rückt (Kapitel 6).

Thema des sechsten Kapitels ist schließlich die Globalisierung und Sinisierung der chinesischen Soziologie der vergangenen Jahrzehnte; zwei Perspektiven, die in der chinesischen Soziologie zu Recht als komplementär gesehen werden. In den Debatten um die Sinisierung der Soziologie, die in den 1980er- und 1990er-Jahren von Diskursen in „Greater China“, insbesondere in Taiwan und Hongkong, geprägt waren, sieht Chen nun die Soziologen und Soziologinnen Festlandchinas als maßgebliche Akteure und Akteurinnen. Debatten zu einer genuin chinesischen Soziologie gehören zwar zu ihrem Erbe (Xiangtu Zhongguo von Fei Xiaotong ist ein Beispiel dafür) und sind schon immer fester Bestandteil der Fachdiskurse, auch nach der Wiedereinführung des Fachs, aber haben aus Chens Sicht bislang meist nur oberflächlich oder vordergründig stattgefunden (S. 135).

Chen Hon Fai gibt einen informativen und spannenden Einblick in die Soziologie in China, wesentlich aus wissenschaftssoziologischer Perspektive. Er nimmt insbesondere die institutionellen Bedingungen in den Blick und damit die prominente Rolle der Institutionen auf die Geschichte und Gegenwart des Fachs. Weiterhin gibt das Buch einen Einblick in wichtige Themen, wissenschaftstheoretische Strömungen und Methodendebatten der Soziologie. Eine deutlichere Trennung dieser beiden Perspektiven in zwei Teile wäre dabei gewinnbringend gewesen. Das gilt auch in Bezug auf die große Zeitspanne, in der sich der Autor hin und her bewegt und die die Orientierung erschwert. 
Funding Open Access funding enabled and organized by Projekt DEAL.

Open Access Dieser Artikel wird unter der Creative Commons Namensnennung 4.0 International Lizenz veröffentlicht, welche die Nutzung, Vervielfältigung, Bearbeitung, Verbreitung und Wiedergabe in jeglichem Medium und Format erlaubt, sofern Sie den/die ursprünglichen Autor(en) und die Quelle ordnungsgemäß nennen, einen Link zur Creative Commons Lizenz beifügen und angeben, ob Änderungen vorgenommen wurden.

Die in diesem Artikel enthaltenen Bilder und sonstiges Drittmaterial unterliegen ebenfalls der genannten Creative Commons Lizenz, sofern sich aus der Abbildungslegende nichts anderes ergibt. Sofern das betreffende Material nicht unter der genannten Creative Commons Lizenz steht und die betreffende Handlung nicht nach gesetzlichen Vorschriften erlaubt ist, ist für die oben aufgeführten Weiterverwendungen des Materials die Einwilligung des jeweiligen Rechteinhabers einzuholen.

Weitere Details zur Lizenz entnehmen Sie bitte der Lizenzinformation auf http://creativecommons.org/ licenses/by/4.0/deed.de.

Dominique Schirmer Dr., akademische Oberrätin am Institut für Soziologie der Albert-Ludwigs-Universität Freiburg. Forschungsschwerpunkte: Internetmedien, empirische Sozialforschung, integrierte Methoden, feministische und ,queerfeministische“ Theorien, China, chinesische Soziologie. Aktuelle Publikationen: Die qualitative Analyse internetbasierter Daten. Methodische Herausforderungen und Potenziale von Online-Medien. Wiesbaden 2015 (hrsg. mit N. Sander u. A. Wenninger); Empirische Methoden der Sozialforschung. Grundlagen und Techniken. Paderborn 2009; Soziologie und Lebensstilforschung in der Volksrepublik China. Perspektiven einer Mikrotheorie gesellschaftlichen Wandels. Bielefeld 2004. 